但无孤立超限数解, 即 $x=\eta$ （因而 $y=\eta^{2}$ ) 不能是 现立超限数。

容易研究 (1) 式的七种变形方程的超限数解， 比如我们有下列定理:

定理 2 方程 $x^{4}=D y^{2}+1$ 没有招限数解.

定王 3 方程 $y^{2} D=x^{4}+1$ 当且仅当 $D=2$ 时有却限序数解。

定王 4 方程 $x^{4}=y^{2} D+1$ 没有超限数解。

我们可以进一步研究方程 $x^{n+2}=1+D y^{*}$ 和 方程 $x^{2 n}=1+D^{n}$, 及它们的十四种变形方程的 超限数解. 比如, 可以得到以下主要结果:

定理 5 方程 $x^{n+2}=1+D y^{n}(D$ 和 $n$ 为自然
数)在 $D=1$ 时有超限序数解。

$$
\left\{\begin{array}{l}
x=\eta^{\mu}, \\
y=\eta^{n+2},
\end{array} \quad \eta\right. \text { 为任一超限数; }
$$

$D \geqslant 2$ 时有极限数解 (3); $D \geqslant 2, n=1$ 时有超限 数解 (可以是孤立数); $D \geqslant 2, n \geqslant 2$ 时没有超限现 立数解.

定理 6 方程 $x^{n+2}=y^{n} D+1$ 在 $n=1$ 时有 超限数解, 在 $n \geqslant 2$ 时无超限数解。

定理 7 方程 $x^{2 n}=D y^{n}+1$ 在 $n=1$ 时有 超限数解, 在 $n \geqslant 2$ 时无超限数解.

胡庆平

(西北大学数学系)

\title{
用脉冲 $\mathrm{He}-\mathrm{Ne}$ 激光的分幅高速干涉摄影装置
}

用于诊断在通明介质环境中发生的快速、短暂 现象的分愊高速千涉振影装置，已在中国科学院力 学研究所研制成功. 它是由脉冲 $\mathrm{He}-\mathrm{Ne}$ 激光光源、 抗强振动的干涉仪及高速振影机三部分组成. 由于 笠单简、抗振珄好、获得的干涉图片结果直观、使 用稳定可茽，能广地用于弨音速风洞流场测量、燃 烧、爆炸及强激光与物质相互作用等的研究中.

该装置的研制中首次提出了逆程序运转方案， 将连续输出的 $\mathrm{He}-\mathrm{Ne}$ 漖光成功地改造成为时间可控 的同步脉冲光源，其原理是预先使 He-Ne 漖光器大 于工作电流运转, 此时不出光. 当同步信号输人后, 切断电源, He-Ne 湤光管的电流迅速下降至工作电 流, 此时输出一脉冲光. 这一实现将连续 $\mathrm{He}-\mathrm{Ne}$ 做 光改造成可控同步脉冲光源的方案是有创造性的. 装管中用了自制的平晶错位干涉仪。这是一种只有 一块干涉元件、可以获得比一般干涉仪视场大、最具 抗振性的干沙仪。它简单、经济、易于调整维护。
研制中还成功地解决了提高干涉光束光强的技 术, 设计了合理的光学匹配光路并解决了脉冲 $\mathrm{He}-$ $\mathrm{Ne}$ 光源、研究现象与高速掫影机三者问的时间同 步, 完善了整个装置. 与转镜分蕾超高速振影机配 合，仅用 1 豪瓦经过改造的脉冲 He-Ne 湤光，得到 了一系列分幅高速干涉炤片，每愊曝光时间 1.3 谈 秒. 其中干涉仪与各种类型高速摄影机均能配合使 用.

该装置在力学所经过一年多的运转考验及技术 鉴定已用于诊断强振动燃烧型超音速风洞流场测量 及强败光与物质相互作用的研究中. 能在一次实验 中同时取得数十幅研究现象发展过程的高速干涉照 片，这对快速、短暂现象的研究提供了有效的观测 手段。

$$
\text { 夏生录 }
$$

(中国科学院力学研究所)

\section{金属离子水解的规律性}

关于金属离子水解程度的规律性, 许多人从离 于极化梳念出发进行了研究。近期，温元凯、甘昌汉 等人提出两个经验公式。然而前者复杂，后者物理 意义不解明确. 由于误差较大，规律性不太明显.

我们在分析已有的金属离子水解常数实验数据 和前人对金属离子水解过程的讨论后，认为金属离 子的水解是由该离子和水分子之间的相互作用而引
起的, 此种作用就是金属离子和水分子之间的极化 作用. 其实质就是金属离子所具有的场力（静电吸 力）对水分子的作用使水分子的结构发生破裂的反 映. 随着金属离子场力的增强，对水分子的极化也 增强,这样，不仅引起水分子的变形，并促使水分子 的结构发生断裂成为两部分，与离子电荷相反的羟 基和离子组合在一起并离解出 $\mathrm{H}^{+}$离子, 因而水解 
常数就大. 然而金属离子的场力是由离子的电荷、 半径和外层电子结构决定的. 故水解常数 $P K_{1}$ 和金 属离子的电荷 $z$ 、半径 $r$ 和外层构型之间就有一定 的函数关系. 基此,提出下式:

$$
P K_{1}=-\left(0.8 \frac{Z^{2}}{r}+0.3 \frac{Z}{r}\right)+A,
$$

式中 $P K_{1} 、 Z 、 r$ 具有通常的意义. $A$ 是和金属离子 外层电子构型有关的常数。对具有情气和 $t^{*} d^{0} s^{0} p^{0}$ 构型的离子为 16.5 ; 具有 $(18)$ 和 $(18+2)$ 构型的 离子为 13.0 ; 具有过渡元素的离子为 14.3 . 半径 $r$
采用 Sanderson 和 Ahrens 的数值.

利用上式计算了 56 种金属离子的水解常数. 对 具有情气和 $f^{*} d^{0} s^{0} p^{0}$ 构型的 33 种金属离子，计算值 和实验值的偏差均在 1.1 以内. 对具有非情气构型 的 23 种金属离子, 计算值和实验值的偏差有 5 个数 据超过了 1.1. 由此看出，尽管该式仍是一种近似， 但较以前的经验公式，更能反映金属高子水解过程 的真实性.

杨进元 (郑州大学化学系)

\section{Arakawa 格式的非线性计算不稳定性 及三种长时间计算稳定的格式}

非线性计算不稳定是数值天气预报中十分突出 的问题，也是计算数学和计算力学等学科中共同关 心的问题. 近两年来, 我们较系统地研究了有关非 线性计算稳定性的若干问题，特别的证明了许多常 见的显式格式 (包括著名的 Arakawa 格式) 都存在 非线性计算不稳定的特例，我们还构造了三种能长 时间计算稳定的格式.

在 $(x, y)$ 空间布网, 令 $F_{i}=\frac{F^{n+1}-F^{n}}{\tau}, \bar{F}_{t}^{i}=$ $\frac{F^{N+1}-F^{n-1}}{2 \tau}, \bar{F}=\frac{F^{N+1}+F^{n}}{2} ; \bar{F}^{x}=\frac{F_{j+\frac{1}{2}, k}+F_{i-\frac{1}{2}, A}}{2}$, $F_{x}=\frac{F_{i+\frac{1}{2}, k}-F_{j-\frac{1}{2}, k}}{h}, \quad F_{+k}=\frac{F_{j+1, k}-F_{i, k}}{h}$,

$$
F_{-*}=\frac{F_{i, k}-F_{i-1, k}}{h} ;
$$

$y$ 方向类似.

对于二维无辐散涡度方程

$$
\frac{\partial \zeta}{\partial t}+u \frac{\partial \zeta}{\partial x}+v \frac{\partial \zeta}{\partial y}=0
$$

的 Arakawa 格式是

$$
\bar{\zeta}_{i}^{\prime}+\frac{1}{3}\left(J^{++}+J^{x+}+j^{+x}\right)=0,
$$

其中 $J^{++}=\bar{\Psi}_{x}^{x} \bar{\zeta}_{y}^{y}-\bar{\Psi}_{y}^{y} \bar{\zeta}_{x}^{x}, J^{x+}=\overline{\left(\Psi \bar{\zeta}_{y}^{y}\right)_{x}^{x}}-\overline{\left(\Psi \tilde{\zeta}_{x}^{x}\right)_{y}^{y}}$,

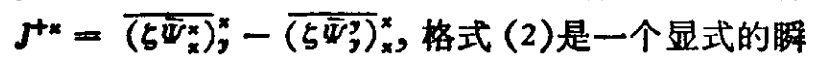

时平方守佰的格式,把

$$
\begin{aligned}
\Psi_{i, k}^{n}= & \left(C n \cos \frac{\pi i}{2}+S n \sin \frac{\pi i}{2}+U_{n} \cos \pi i\right) \\
& \times \sin \frac{2 \pi}{3} k,
\end{aligned}
$$

代人 (2) 式,可证 Arakawa 格式同样会出现非线珄 计算不稳定.

利用“共辑内积法”, 由(1)式我们构造了如下三 种隐式格式:

$$
\begin{aligned}
& \zeta_{+t}+\frac{1}{2}\left[u^{*} \bar{\zeta}_{+*}+\left(u^{*} \bar{\zeta}\right)_{-*}+v^{*} \bar{\zeta}_{+g}+\left(v^{*} \bar{E}\right)_{-3}\right] \\
& =0 \text {, } \\
& \zeta_{++}+\frac{1}{2}\left[u^{*} \bar{\zeta}_{-*}+\left(u^{*} \bar{\zeta}\right)_{+*}+v^{*} \bar{\zeta}_{-y}+\left(v^{*} \bar{\xi}\right)_{++}\right] \\
& =0 \text {, } \\
& \xi_{+t}+\frac{1}{2}\left[u^{*} \bar{\xi}_{x}^{x}+\overline{\left(u^{*} \bar{\zeta}\right)_{x}^{x}}+v^{*} \bar{\xi} y+\overline{\left(\nu^{*} \bar{\xi}\right)}, y\right] \\
& =0 \text {, }
\end{aligned}
$$

其中 $u^{*}, v^{*}$ 是 $u, v$ 的某种光滑值, 不难验证上述 三个格式具有时空差分意义下的总涡度的平方守恒 性，因此它们是绝对稳定的,其中 (6) 式就是曾庆存 和季仲贞早在 1964 年就已构造的完全平方守佰的 隐式格式在涡度方程上的体现。

季仲贞

（中国科学院大物理研究所） 